\title{
THE PAULI EXCLUSION PRINCIPLE, SPIN, AND STATISTICS IN LOOP QUANTUM GRAVITY: $S U(2)$ VERSUS $S O(3)^{*}$
}

\author{
JOHN SWAIN \\ Department of Physics, Northeastern University, Boston, MA 02115 \\ e-mail: john.swain@cern.ch
}

\begin{abstract}
Recent attempts to resolve the ambiguity in the loop quantum gravity description of the quantization of area has led to the idea that $j=1$ edges of spin-networks dominate in their contribution to black hole areas as opposed to $j=1 / 2$ which would naively be expected. This suggests that the true gauge group involved might be $S O(3)$ rather than $S U(2)$. We argue that the idea that a version of the Pauli principle is present in loop quantum gravity allows one to maintain $S U(2)$ as the gauge group while still naturally achieving the desired suppression of spin- $1 / 2$ punctures. Such an idea can be motivated by arguments from geometric quantization even though the $S U(2)$ under consideration does not have the geometrical interpretation of rotations in 3-dimensional space, and its representation labels do not correspond to physical angular momenta. In this picture, it is natural that macroscopic areas come almost entirely from $j=1$ punctures rather than $j=1 / 2$ punctures, and this is for much the same reason that photons lead to macroscopic classically observable fields while electrons do not.
\end{abstract}

\section{Introduction}

This talk is based on an essay which received an an honorable mention in the 2003 Essay Competition of the Gravity Research Foundation [1, and its subsequent and significant amplification 2 .

It is a contribution to trying to figure out what the rules should be for a theory of quantum gravity in $3+1$ dimensions based on a nonperturbative treatment in the framework now generally referred to as "loop quantum gravity". The successes of this approach using the Ashtekar variables have been numerous and significant and include proofs that area and volume operators have discrete spectra, and a derivation of black hole entropy up to an overall, yet undetermined constant $\underline{3}$. An excellent recent review leading directly to this paper is by Baez 4 , and its influence on this introduction will be clear.

One thinks of a spin-network as floating freely, but able to intersect physical surfaces, with each edge contributing to its area. To a good approximation, the area $A$ of a surface which intersects a spin network at $i$ edges, each carrying an $S U(2)$ label $j$ is given in geometrized units (Planck length equal to unity) by

\footnotetext{
*Talk at the 10th Marcel Grossmann Meeting in Rio de Janeiro, 20-26 July 2003
} 
$A \approx \sum_{i} 8 \pi \gamma \sqrt{j_{i}\left(j_{i}+1\right)}$, where $\gamma$ is the Immirzi-Barbero parameter $\underline{\text { [5] }}$. The most important microstates consistent with a given area are those for which $j$ is as small as possible, which one would expect to be $j_{\min }=1 / 2$. In this case, each contribution to the area corresponds to a spin $j=1 / 2$ which can come in two possible $m$ values of $\pm 1 / 2$. For $n$ punctures, we have $A \approx 4 \pi \sqrt{3} \gamma n$ and entropy $S \approx \ln \left(2^{n}\right) \approx \frac{\ln (2)}{4 \pi \sqrt{3} \gamma} A$.

Now if we use Hawking's formula $\sqrt{6}$ for black hole entropy $S=A / 4$ to get $\gamma=$ $\frac{\ln (2)}{\pi \sqrt{3}}$ and the smallest quantum of area is then $8 \pi \gamma \sqrt{\frac{1}{2}\left(\frac{1}{2}+1\right)}=4 \ln (2)$. A black hole's horizon then acquires area, to a good approximation, from the punctures of many spin network edges, each carrying a quantum of area $4 \ln (2)$ and one "bit" of information, in accordance with Wheeler's "it from bit" philosophy 7 .

Bekenstein's early intuition $\sqrt{8}$ that the area operator for black holes should have a discrete spectrum made of equal area steps (something not really quite true in loop quantum gravity in full generality) was followed by Mukhanov's observation 9 that the $n^{\text {th }}$ area state should have degeneracy $k^{n}$ with steps between areas of $4 \ln (k)$ for $k$ some integer $\geq 2$ in order to reproduce Hawking's expression $S=A / 4$. For $k=2$ one would have the $n^{\text {th }}$ area state described by $n$ binary bits.

On the other hand Hod ${ }^{10}$ has argued that by looking at the quasinormal damped modes 11 of a classical back hole one should be able to derive the quanta of area using the formula $A=16 \pi M^{2}$ relating area and mass of a black hole to get $\Delta A=32 \pi M \Delta M$ for the change in area accompanying an emission of energy $\Delta M$. Nollert's computer calculations 12 of the asymptotic frequency $\omega$ of the damped normal modes gave $\omega \approx 0.4371235 / M$, so setting $\omega=\Delta M$ one finds $\Delta A \approx 4.39444$, which one might guess is exactly $4 \ln (3)$. Motl 13 showed that this is indeed the case.

One might expect $\frac{14}{14} \approx 4 \ln (3)$ instead of $\Delta A \approx 4 \ln (2)$ if the spin network edges contributing to the area of a black hole didn't carry $j=1 / 2$, but rather $j=1$. In this case $j_{\min }$ would be 1 rather than $1 / 2$, there would be three possible $m$ values, area elements would be described not by binary "bits", but by trinary "trits" (see also 15) and it suggests that the correct gauge group might be $S O(3)$ rather than $S U(2)$.

So which is it? Corichi has argued 16 that one might arrive at the conclusion that $j_{\min }=1$ by thinking of a conserved fermion number being assigned to each spin$1 / 2$ edge. The exact physical interpretation of this is not entirely clear, but roughly one might imagine that there is a sort of conserved fermion number which would disallow a single spin network edge from leaving a surface and being left "dangling in the bulk". Ling and Zhang $\frac{17}{17}$ have argued that going to $N=1$ supergravity would also offer a way to avoid spin-1/2 edges.

\section{An Exclusion Principle?}

The point of the original essay for the Gravity Research Foundation was to suggest that if one assumes (following Pauli and real spins) that no more than one $j=1 / 2$ 
edge (think of "one spin-1/2 particle") would allowed to puncture a surface (think of "occupy a given quantum state"), then everything makes sense. How can one justify such a conjecture?

In quantum mechanics, the spin-statistics theorem is simply a postulate: as Dirac ${ }^{18}$ puts it "to get agreement with experiment one must assume that two electrons are never in the same state" (my italics). If one is honest, the usual quantum field theory arguments are not much better. The requirement that field operators should commute at spacelike separations which seems reasonable if one wants appropriate notions of causality and locality comes to grief for fermions (i.e. one has an unstable vacuum). Jordan's anticommutators 19 save the day, but obscure the connection to causality unless one assumes that the fermion fields are Grassmann-valued. A rather comprehensive review of the history and literature is in 20

For a surface punctured by spin network edges I want to argue that one should consider an amplitude which returns to its original value, up to a phase, upon the exchange of two spin-1/2 (and thus identical, indistinguishable) punctures. If making the exchange twice leads to the identity, one then needs merely to choose a sign, and -1 seems at least as natural as +1 . This argument can be sharpened in the following way:

For a configuration space of $n$ spin- $j$ non-coincident identical punctures, as shown long ago by Laidlaw and DeWitt $[21$, phases for propagators must form a scalar unitary representation of the fundamental group. This limits the possible choice of statistics to Bose statistics (no phase change under permutations) or Fermi statistics (change of sign for any odd permutation). As it stands, this is just a statement about what statistics are possible, but has nothing to do with rotations, $S U(2)$, $S O(3)$, or even physical space.

Now to argue for Fermi statistics for odd half-integer $j$ punctures and Bose statistics for integer $j$ punctures we cannot use the usual QFT arguments as we have no background spacetime. The same applies to heuristic arguments based on identifying an exchange as a composition of physical rotations (i.e. 22 ) and to arguments based on extended kink-like objects (i.e. 23 ), since the punctures are meant to be points. Related approaches such as those of Balachandran et al. 25, Tseuschne1 24, Berry and Robbins 26 , and many others in 20 all seem difficult to apply. For an argument not dependent on a prior concept of physical space we look directly at the configuration space and use ideas from geometric quantization 27 , following beautiful arguments of Anastopoulos 28 .

Consider the quantization of the sphere $S^{2}=\left\{\left(x_{1}, x_{2}, x_{3}\right) \mid x_{1}^{2}+x_{2}^{2}+x_{3}^{2}=1\right\}$ with the symplectic form $\Omega=\frac{1}{2} s \epsilon_{i j k} x^{i} d x^{j} \wedge d x^{k}$ and a symplectic action of $S O(3)$ on $S^{2}$ where $S O(3)$ acts on the $x^{i}$ in the usual way by its defining representation and obviously leaves $\Omega$ invariant. Each choice of $s$ gives a different symplectic manifold, and the requirement that $\Omega$ be integrable requires that $s=n / 2$ with $n$ an integer. In this way $s$ corresponds to the usual notion of spin in quantum mechanics. Note 
that so far there is no explicit identification of the $x^{i}$ with spacetime directions they just happen to define the coordinates on an abstract $S^{2}$. We also have the $U(1)$ bundle provided by the Hopf map from $S^{3}$ to $S^{2}$ defined by $\pi(\xi)^{i}=\bar{\xi} \sigma^{i} \xi$, defined in terms of 2-component complex "spinors" $\xi$ of length 1 and $\sigma^{i}$ the Pauli matrices. $\pi(\xi)^{i}$ is obviously real and invariant under $\xi \rightarrow u \xi$ for any $u=\exp (i \theta)$ in $U(1)$. For $s=1 / 2$ we have $U(1)$ operations corresponding to the two square roots of unity \pm 1 , both of which correspond to the identity element of $S O(3)$.

A prequantization is given by the bundle whose total space is the set of orbits of $S^{3}$ under the two $U(1)$ actions of multiplication by \pm 1 and the same projection map $\pi$. $S U(2)$ actions on $S^{3}$ correspond to $S O(3)$ actions on $S^{2}$, and we pick up a factor of -1 for a $2 \pi$ rotation. We can now think of an $s=1 / 2$ state as a point on the sphere $S^{2}$, accompanied by this sign change for $2 \pi$ rotations. Now if we make this construction twice out of two classical phase spaces, but assume that the two are the same $S^{2}$, we can exchange two points on the $S^{2}$ ("two spins") by two $S O(3)$ rotations, one acting on each point in the same $S^{2}$. For spin- $1 / 2$ this gives a factor of -1 on exchange, and for general spin- $j$ the expected spin-statistics connection 2 .

In other words, even though one might colloquially speak of the edges as carrying "spins", knowing full well that this is really a way of saying " $S U(2)$ representation labels with no obviously necessary connection to spin of elementary particles or irreducible representations of the rotation group in physical space", in fact it does make sense to think of them as physical spins and argue for a spin-statistics theorem. In this sense the spin-statistics theorem might better be thought of as a sort of " $(S U(2)$ representation label)-(sign change or not on exchange)" theorem.

In loop quantum gravity this leads then to a picture in which a large black hole does receives contributions to its area from spin- $1 / 2$ (and spin- $3 / 2$, spin- $5 / 2$, etc.) punctures, but these are always very small compared to the enormous number of $j=1$ edges. The value $j=1$ is the lowest value of $j$ contributing nonzero area not being severely limited by Fermi-Dirac statistics, and able to appear arbitrarily often. This can then make it look like we're dealing with $S O(3)$ rather than $S U(2)$.

\section{Conclusions}

In a sense, the question of $S U(2)$ vs. $S O(3)$ in loop quantum gravity could be very much like one that we face in everyday physics. Integer spin particles, which fall into $S O(3)$ representations, obey Bose-Einstein statistics and gregariously bunch together to give large macroscopically observable fields such as electromagnetic fields. Half-integer spin particles do not. We could well be excused for thinking that the symmetry group of our world under rotations was $S O(3)$ rather than $S U(2)$. Indeed, until the discovery of spin, it did appear that physical rotations were always elements of $S O(3)$. The need for $S U(2)$ was, in many ways, a surprise!

These ideas are likely hard to test, but it is possible to make some predictions. For example, the $S U(2)$ theory with the exclusion principle proposed here will give both: a) what seems to be the correct result for large black holes, with areas 
well-described by values which go up in steps of $4 \ln (3)$; and b) the possibility of simultaneously allowing areas as small as $4 \ln (2)$.

\section{Acknowledgments}

It is a great pleasure to thank the session and conference organizers, especially Carlo Rovelli (via email), Robert Oeckl, Santiago Perez Bergliaffa, Mario Novello, and Remo Ruffini for their help and hospitality, and everyone in Rio, the most beautiful city in the world! Muito obrigado!

\section{References}

1. J. Swain, http://arXiv.org/abs/gr-qc/0305073

2. J. Swain, Int. J. Mod. Phys. D (2003) 1729.

3. A. Ashtekar and J. Lewandowski, Class. Quant. Grav 14 (1997) A55; C. Rovelli and L. Smolin, Nucl. Phys. B442 (1995) 593, Erratum-ibid. B456 (1995) 753.

4. J. Baez in "Matters of Gravity, the newsletter of the APS Topical Group on Gravitation, Spring 2003", J. Pullin (ed.), http://arXiv.org/abs/gr-qc/0303027.

5. A. Ashtekar et al., Phys. Rev. Lett. 80 (1998) 904; G. Immirzi, Nucl. Phys. Proc. Suppl. 57 (1997), 65. F. Barbero, Phys. Rev. D51 (1995), 5507.

6. S. Hawking, Commun. Math. Phys. 43 (1975), 199.

7. J. Wheeler, in Sakharov Memorial Lecture on Physics, vol. 2, eds. L. Keldysh and V. Feinberg, Nova Science, New York, 1992.

8. J. Bekenstein, Phys. Rev. D7 (1973), 2333.

9. V. Mukhanov, JETP Lett. 44 (1986), 63; J. Bekenstein and V. Mukhanov, Phys. Lett. B360 (1995), 7.

10. S. Hod, Phys. Rev. Lett. 81 (1998), 4293;S. Hod, Gen. Rel. Grav. 31 (1999), 1639.

11. V. Cardoso, J. P. S. Lemos, S. Yoshida, Phys. Rev. D69 (2004) 044004.

12. H.-P. Nollert, Phys. Rev. D47 (1993), 5253.

13. L. Motl, Adv.Theor.Math.Phys. 6 (2003) 1135-1162.

14. O. Dreyer, Phys. Rev. Lett. 90 (2003) 081301.

15. L. Motl and A. Neitzke, Adv. Theor. Math. Phys. 7 (2003) 307.

16. A. Corichi, Phys. Rev. D67 (2003) 087502.

17. Y. Ling and H. Zhang, Phys. Rev. D68 (2003) 101501.

18. P. A. M. Dirac, "The Principles of Quantum Mechanics (4th ed.)", Oxford University Press, 1958.

19. P. Jordan, Zeitschrift für Physik, 44 (1927) 473.

20. I. Duck and E. C. G. Sudarshan, "Pauli and the Spin-Statistics Theorem", World Scientific, 1997.

21. M. G. G. Laidlaw and C. Morette DeWitt, Phys. Rev. D3 (1971) 1375.

22. E. Donth, Physics Letters 32A (1970) 209.

23. D. R. Finkelsetein and J. Rubensetin, J. Math. Phys. 9 (1968) 1762.

24. R. Tscheuschner, Int. J. Theor. Phys. 28 (1989) 1269, erratum Int. J. Theor. Phys. 29 (1990) 1437.

25. A. P. Balachandran, A. Daughton, Z. C. Gu, G. Marmo, R. D. Sorkin and A. M. Srivastava, Mod. Phys. Lett. A5 (1990) 1575; Int. J. Mod. Phys. A8 (1993) 2993.

26. M. V. Berry and J. M. Robbins, Proc. Roy. Soc. Lond. A453 (1997) 1771; J. Phys. A33 (2000) L207.

27. D. J. Simms and N. M. J. Woodhouse, "Lectures on Geometric Quantization", Springer Lecture Notes in Physics 53, Springer-Verlag, 1977; N. M. J. Woodhouse, "Geometric Quantization (2nd ed.)", Oxford University Press, 1991; J.-M. Souriau, "Structure of Dynamical Systems", Birkhäuser Press, Boston, 1997. 
28. C. Anastopoulos, http://arXiv.org/abs/quant-ph/0110169 . 